\title{
A review of a motion model construction and simulation method for multi-level and multi-dimensional dynamic systems
}

\author{
Guoxin Yang* \\ Shanghai Jiao Tong University \\ Ji Yang ${ }^{\dagger}$ \\ University of Illinois, Urbana Champaign
}

(Dated: November 13, 2022)

\begin{abstract}
On October 21, 2020, the invention "A multi-level and multi-dimensional dynamic system motion model construction and simulation method" was accepted by the State Intellectual Property Office of China [2]. Through the construction and simulation of the motion model of a multi-level and multidimensional dynamic system, the motion trajectory of the motion factors in each dynamic system is nested and substituted into the next dynamic subsystem to establish the motion trajectory of the motion factors in its dynamic subsystem. Until the dynamic trajectory model of the motion factors in the minimum dynamic subsystem is obtained, the motion model of a multi-level and multi-dimensional dynamic system is constructed, which reveals the motion law that can unify the microscopic particles and all the motion factors in the macroscopic universe. Multiple dynamic systems are entangled systems. If the entanglement properties are obtained not by "absolutely simultaneous" measurements of the entangled particles, it just proves that there is no non-local hyper-distance action.
\end{abstract}

\section{INTRODUCTION}

The universe and all things move, interact and change in a multi-level and multi-dimensional dynamic system. On the macro level, from galaxy groups, galaxies, stars and planets to the various and ever-changing universe, all are dynamic systems at different levels. On the micro level, from molecules, atoms, protons and neutrons to electrons, neutrinos, quarks, gluons and other basic particles to energieelements, all are dynamic systems of different levels.

\section{DESCRIPTION OF MOTION LAW OF THE MULTI-LEVEL AND MULTI-DIMENSIONAL DYNAMIC SYSTEM}

An embodiment of the invention is presented as follows, which describes a motion model construction method for a multi-level and multi-dimensional dynamic system proposed by the invention.

1. The structure of the dynamic subsystem $S_{2}$ and its composition of the dynamic factors are analyzed for any original dynamic system $S_{1}$.

2. The above analysis process is repeated, and the obtained dynamic subsystem $S_{2}$ is analyzed until the $S_{n}$ structure and dynamic factor composition of the minimum dynamic subsystem constituted by the dynamic system are obtained.

\footnotetext{
*yangguoxinsc@abchina.com

$\dagger$ jiyang3@illinois.edu
}

3. The motion trajectory model is established of the motion factor in the dynamic system, taking the motion factor of the original dynamic system and using the computer according to the composition of the dynamic factors driving the motion factor.

4. The process is repeated of establishing the motion trajectory model, and the motion trajectory of the motion factor in each dynamic system is nested into the next dynamic subsystem to establish the motion trajectory of the motion factor in the dynamic system, until the motion trajectory model is obtained of the motion factor in the minimum dynamic subsystem.

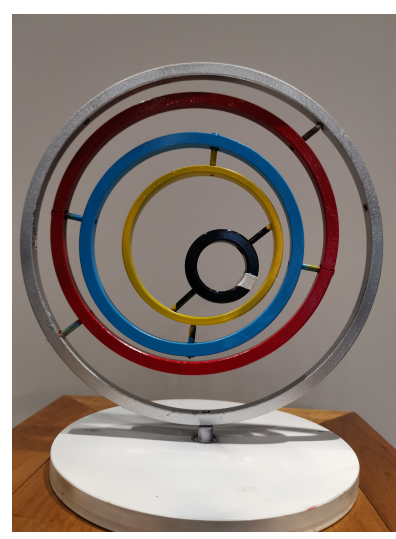

(a)Model in static

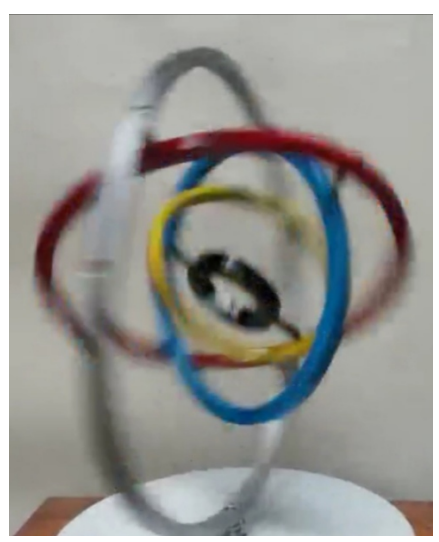

(b)Model in motion
FIG. 1. Real-world multi-level and multi-dimensional dynamic system model.

As shown in the process above, in this embodiment, a multi-level and multi-dimensional dynamic system mo- 
tion model construction method is presented, which includes the following steps: The structure of the dynamic subsystem $S_{2}$ and its composition of the dynamic factors are analyzed for any original dynamic system $S_{1}$. The above analysis process is repeated, and the obtained dynamic subsystem $S_{2}$ is analyzed until the $S_{n}$ structure and dynamic factor composition of the minimum dynamic subsystem constituted by the dynamic system are obtained.

Taking the motion factor of the original dynamic system, the motion trajectory model of the motion factor in the dynamic system is established according to the composition of the dynamic factors driving the motion factor. The process of establishing the above motion trajectory model is repeated, and the motion trajectory of the motion factors in each dynamic system is nested and substituted into the next dynamic subsystem to establish the motion trajectory of the motion factors in its dynamic subsystem, until the motion trajectory model of the motion factors in the minimum dynamic subsystem is obtained.

This multi-level and multi-dimensional dynamic system motion model construction and simulation method, the multi-level and multi-dimensional dynamic system motion model construction process: the original dynamic system $S_{1}$, the dynamic subsystem $S_{2} \ldots$, the dynamical subsystem $S_{n}$ are all spherical systems.

In this embodiment, as shown in Figure 1, it is the concrete model of the five-fold ball dynamic system. The concrete model is reduced to a five-fold circle.

In this embodiment, the construction of the above fivefold sphere dynamic system is provided as follows: A rotation model of a quintuple sphere is built as follows. The first spin ball has radius $\mathrm{R} 1$ and period $T_{1}$. In the first spin sphere, there is a second spin sphere with a radius of $R_{2}$ and a period of $T_{2}$. In the second spin sphere, a third spin sphere is set with a radius of $R_{3}$ and a period of $T_{3}$. In the third rotation ball, the fourth rotation ball is set with a radius of $R_{4}$ and a period of $T_{4}$. The fourth spin ball is set to have the fifth spin ball with radius $R_{5}$ and period $T_{5}$. The centers of the quintuple do not overlap.

The five-fold sphere rotates 15 to 45 degrees apart from each other as it begins to spin. The rotation motion model of a five-fold sphere built by the computer is shown in Figure 3 (a) [4]. When the five-fold sphere is hidden, the rotation motion of the five-fold circle is shown in Figure $3(\mathrm{~b})$. Each point is marked on the five-fold circle, and the rotation motion of the five-fold circle is shown in Figure 3 (c) [5]. The multiple trajectories of the rotational motion of the multiple dynamic systems are shown in Figure 3 (d) [6].

In the method of the multi-level and multi-dimensional dynamic system motion model construction and simulation, the dynamic factors include the dynamic factors provided by the motion factor itself and other dynamic factors provided by the motion factor under its dynamic system.

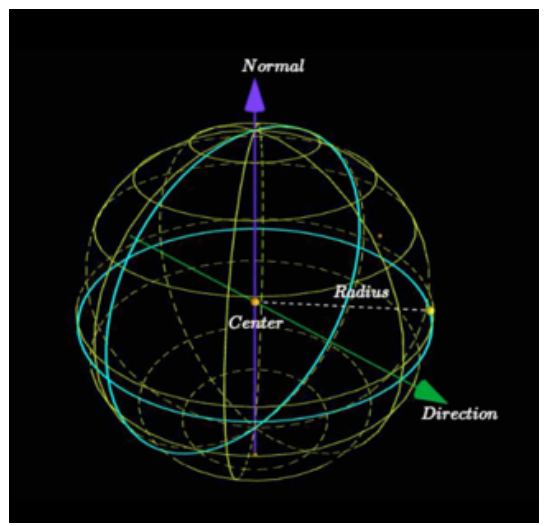

FIG. 2. The mathematical model of the dynamic system.

As shown in Figure 2, in this method of multi-level and multi-dimensional motion model construction and simulation for the dynamic system, the trajectory model of the dynamic system includes the motion trajectory expression of the motion factor consisting of the center of the sphere, normal direction, and radius under the dynamic system:

$$
\begin{aligned}
& \text { sphere }=\{\text { center }:(x, y, z), \\
& \text { norm }:(x, y, z), \\
& \text { direction }:(x, y, z), \\
&\text { radius }: c\},
\end{aligned}
$$

where $x, y$ and $z$ are coordinates of three-dimensional space respectively; $c$ is the default value for the radius.

In this method of motion model construction and simulation of a multi-level and multi-dimensional dynamic system, the motion trajectory of motion factors in each dynamic system is nested and substituted into the next dynamic subsystem to establish the motion trajectory of motion factors in its dynamic subsystem, including the following steps:

- The dynamic trajectory model of the motion factor in the minimum dynamic system Sn with respect to the preset first origin is simulated by computer. Obtain the dynamic trajectory model of the minimum dynamic subsystem $S_{n}$ as the motion factor of its upper layer dynamic subsystem $S_{n-1}$ with respect to the preset 2 origin.

- The above simulation process is repeated to obtain the dynamic trajectory model of the uppermost layer dynamic system $S_{1}$ with respect to the preset nth origin.

- All the obtained dynamic trajectory models are displayed in the three-dimensional space to the multilevel and multi-dimensional dynamic system motion model. 
As shown in the process above, this embodiment provides a step flow diagram of a motion model simulation method of a multi-level and multi-dimensional dynamic system. The motion model simulation of the multidimensional dynamic system includes the following steps:

The dynamic trajectory model of the motion factor in the minimum dynamic subsystem $\mathrm{Sn}$ with respect to the first preset origin is simulated by computer. The dynamic trajectory model is obtained of the minimum dynamic subsystem Sn as the motion factor of its upperlayer dynamic subsystem Sn-1 with respect to the preset 2 origin.

The above simulation process is repeated to obtain the dynamic trajectory model of the uppermost layer power system $S_{1}$ with respect to the preset nth origin.

All the obtained dynamic trajectory models are displayed in three dimensional space to obtain a multi-level and multi-dimensional dynamic system motion model.

In this embodiment, a mathematical method for simulating the motion model of a multi-level and multidimensional dynamic system is also provided.

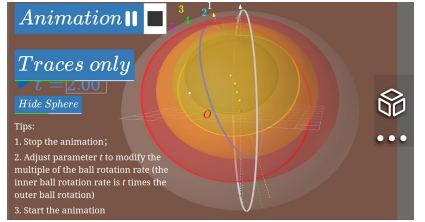

(a)

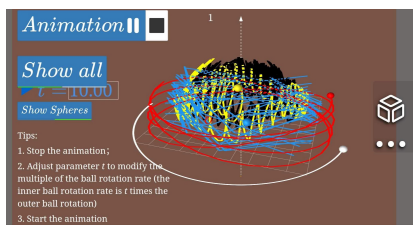

(c)

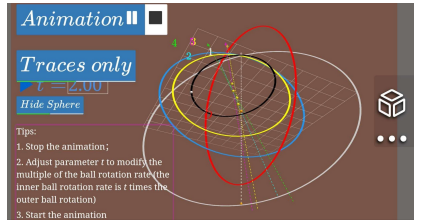

(b)

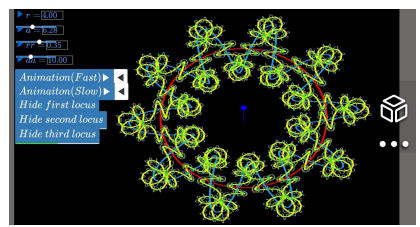

(d)
FIG. 3. Computer simulated multi-level and multidimensional dynamic system model.

Suppose a dynamic system with $n$ layers, the outermost layer is 1 , and the smallest (lowest) layer is $n$. In the mathematical description, the center of the rotating motion dynamical system of the first layer is taken as the origin of coordinates, and the plane rotating through the origin is taken as the base plane. On the base plane are the $X$-axis and $Y$-axis; the $Z$-axis is perpendicular to the base plane, and the $X Y Z$ axis constitutes the three-dimensional space coordinate system of the multidimensional dynamic system. The dynamic system $\left(n_{i}\right)$ of each layer takes the orbital line of the dynamic system $\left(n_{i-1}\right)$ of the next layer as the origin, and the initial angle (also called inclination angle) between the rotating surface and the foundation plane of the first dynamical system is $\theta_{i}$. The rotation radius of the $i$ th dynamical system is the distance $R_{i}$ between its origin and the origin of the $(i+1)$ th dynamic system, the rotational motion velocity (or period) of the dynamic system of $i$ th layer is
$T_{i}$. Thus, the motion trajectory of each dynamic system is shown in Figure 4.

The trajectory of the first dynamic system is in the plane $X Y$ and passing through the origin $O$, with radius $R_{1}$, inclination 0 and operating period $T_{1}$. The motion trajectory of the first dynamical system is $\xi_{1}\left(R_{1}, \theta_{1}, T_{1} \mid 0\right)$, that is $\xi_{1}\left(R_{1}, 0, T_{1} \mid 0\right)$.

When the second dynamic system rotates along the first orbit, it also rotates itself. Its rotation surface has a dip angle of $\theta_{2}$ with $X O Y$ at the beginning, the radius of rotation motion is $R_{2}$, and the period of rotation motion is $T_{2}$. Then the motion trajectory of the second power system is $\xi_{2}\left(R_{2}, \theta_{2}, T_{2} \mid \xi_{1}\right)$. Thus the trajectories of the first and second dynamic systems are iteratively merged into the second dynamic system.

By the same notions, the dynamic system of $i$ th layer rotates along the trajectory line of the dynamic system of the $(i-1)$ th layer, and the radius of its rotation surface is $R_{i}$, the rotation surface has a dip angle of $\theta_{i}$ with $X O Y$ at the beginning, and the rotation motion period is $T_{i}$. Then the operating orbit of the dynamical system of the $i$ th layer is $\xi_{i}\left(R_{1}, \theta_{i}, T_{i} \mid \xi_{i-1}\right)$. Here $\xi_{i-1}$ means the original of the dynamic system of the $i$ th layer is rotating along the trajectory line of the dynamic system of the $(i-1)$ th layer.

If the multi-level and multi-dimensional dynamic system has $n$ layers, the trajectory line of the lowest layer (or internal minimum layer is $n$ ) is $\xi_{n}\left(R_{n}, \theta_{n}, T_{n} \mid \xi_{n-1}\right.$ ), which iteratively merges the trajectory lines of all dynamic systems of $n$ layers into the trajectory line of the $n$th layer.

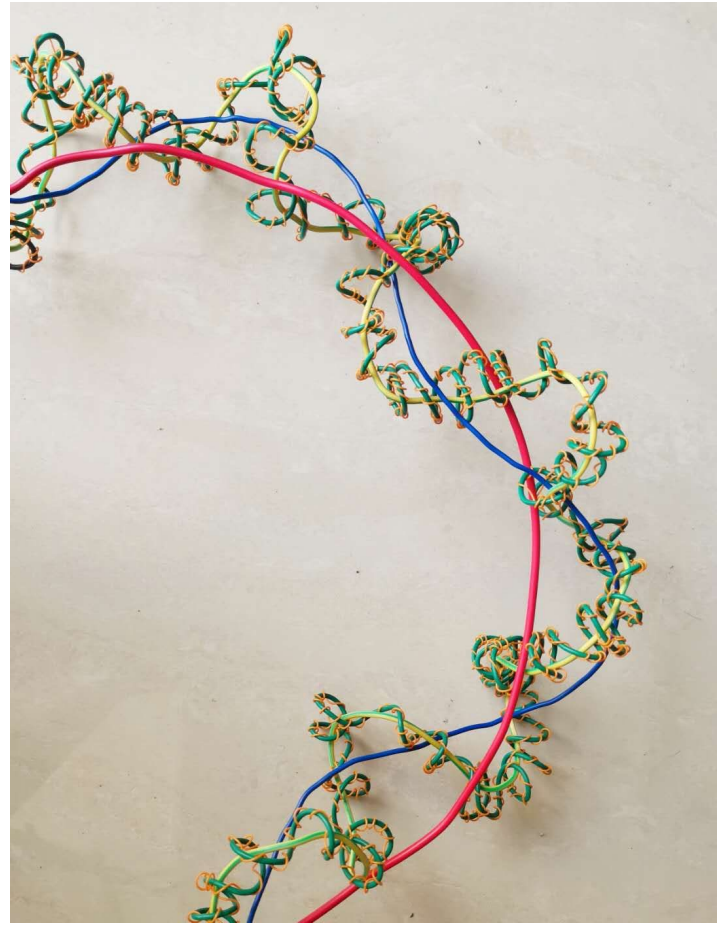

FIG. 4. The motion trajectory of the dynamic system. 


\section{THE MULTI-LEVEL AND MULTI-DIMENSIONAL DYNAMIC SYSTEM IS THE BASIC STRUCTURE AND MOTION LAW OF THE UNIVERSE.}

The multi-level and multi-dimensional dynamic system based on the above construction and simulation can unify the motion law of microscopic particles and all motion factors in the macroscopic universe [3].

\section{Higher dimensional space-time and multiverse}

It is easy to know and understand higher dimensional space-time and multiverse [7] by replacing them with multi-level and multi-dimensional dynamic systems. From the point of view of multi-level and multiple-level dynamic systems, from the Milky Way galaxy, solar system, earth, atom, proton (neutron) to elementary particle are all dynamic systems of different levels, and are also inertial motion systems of different levels. If each dynamic system is defined as one space-time dimension, then a multi-level and multi-dimensional spacetime system, also called a multi-universe, is formed from macro to micro. The space-time dimension composed of multiple dynamic systems is a higher-dimensional spacetime system beyond the current four-dimensional spacetime. Phenomena and problems beyond four dimensional space-time systems can be scientifically understood and explained in higher dimensional space-time systems.

\section{Spatiotemporal curvature [8] is represented in multi-level and multi-dimensional dynamic systems}

Because each dimension of space-time (each rotating dynamic system) has different size, different rotation direction, and different motion time (period or frequency), they determine different space-time characteristics. Therefore, the space-time formed by the dynamic system with different multiplicity has different spacetime characteristics. The space-time formed by different multi-level and multi-dimensional dynamic systems also has different space-time characteristics. The spacetime of these different characteristics is the difference of space-time in different regions of the universe, and the difference of space-time in different regions is manifested as the curvature of space-time. As it travels through the universe, the radiation of light will pass through many different dynamic systems, that is, through space-time of many different properties, and as it passes through spacetime of these different properties, it will be subjected to its effects and produce different changes. As the light passes through a powerful dynamic system, for example, passing through a layer of water vapor, passing through a prism and so on, it will change in a different way. When radiated energy, such as light, passes through a dynamic system with multi-levels or through several dynamic systems, they will act on it and leave a corresponding trace.

\section{Multi-dimensional spatial and temporal characteristics of multi-level and multi-dimensional dynamic systems [1]}

From the macro perspective, everyday things are the first dynamic system, including animals, plants, atmosphere, oceans, etc. For example, the movement changes of the atmosphere show the characteristics of space-time, such as wind and waves, rain or shine and snow. The rotation of the earth is the second dynamic system, and its motion is characterized by 24-hour period, alternation of days and nights and other spatiotemporal characteristics. The earth's revolution (that is, the rotation in the solar system) is the third dynamic system, and its motion changes show the spatiotemporal characteristics such as the 365-day cycle and the alternation of spring, summer, autumn and winter seasons. The motion of the solar system around the center of the Milky Way galaxy is the fourth dynamic system, and its motion in the Milky Way galaxy is characterized by the period of 226 million years and the climate change over tens of millions of years [3]. The motion of the Milky Way galaxy in the galaxy cluster forms the fifth dynamic system [9]. Too little is known or experienced of the temporal and spatial properties of the fifth dynamic system, just like the extinction of the dinosaurs, human beings are only 5 million years old now. Hundreds of millions of years later, human beings may disappear like the dinosaurs. In this way, human beings will never understand or experience the spatiotemporal characteristics of the fifth dynamic system.

From the microscopic perspective, the molecules of the object are the first dynamic system. Molecules determine the basic properties of an object, such as the density (specific gravity), hardness, temperature, solid, liquid, gas and other different states. These basic properties are the space-time properties of the molecular level. The atom is the second dynamic system. The periodic nature of its motion (as listed in the periodic table) is the spacetime nature of the atomic level. Subatomic particles such as protons and neutrons are the third dynamic system. Their motion period and motion mode are the spacetime characteristics of the subatomic level. Electrons, quarks and bosons are the fourth dynamic system, and their motion period and mode (such as quantum properties) are the space-time properties of elementary particles. The energieelement of elementary particles is the fifth dynamic system [10]. At present, the structure and motion law of this hierarchical dynamical system are not clear. The world's most powerful accelerator, the Large Hadron Collider (LHC) at CERN, has an energy scale of $10^{13}$ electron volts. To detect the structure and motion of energy quanta requires a high energy collider of $10^{24}$ to $10^{28}$ electron volts, it would require building colliders that are between a trillion kilometers and 1,000 lightyears long, which would be too difficult for humans to 
handle. Humans may have disappeared long before they were finished, and in that sense it is impossible to detect the structure and motion of a quantum.

\section{Quantum effects [11] being the basic law of multi-level and multi-dimensional dynamic systems}

The uncertainty (randomness and superposition) of quantum motion is the essential property of quantum motion in multi-level and multi-dimensional dynamic systems. There are multiple dynamic systems on the quantum. Since each dynamic system has different space-time characteristics (size, direction, and period), the quantum motion follows the motion characteristics (law) of each dynamic system on the quantum. Since quantum simultaneously has the motion characteristics of multi-level and multi-dimensional dynamic systems (magnitude, direction, period), the motion trajectory of quantum must be the superposition and combination of the motion trajectories of the multi-level and multi-dimensional dynamic systems in which it is located. Although the trajectory (property) of each dynamic system is definitely measurable, the motion of multiple dynamic systems is not definitely measurable when combined. In other words, it is impossible to observe the direction and position and velocity of quantum motion at any given moment from outside the multiple dynamic systems. Look at the central track in Figure 3 (c). To measure or predict quantum motion from different dynamic systems, different results are bound to be obtained. Therefore, the uncertainty, randomness and superposition state of quantum motion are the essential properties of the operation law of multi-level and multi-dimensional dynamic systems.

The motion of the quantum described should be realized along the superposition (iteration) of the motion of the multilevel dynamic systems to reveal its true process, as depicted in Figure 3 (d) through successive iterations.

In fact, if you look at the motion of every person on Earth from outside the galaxy cluster, it would also take on a quantum state. Quantum effects are not understood because we now observe the motion of microscopic particles in multiple dynamic systems only from the four dimensions (from the earth and its four dimensions), rather than from the iteration of multiple dynamic system. It is just as impossible to understand phenomena in three dimensions as it is to observe them only in two dimensions.

The multi-level and multi-dimensional dynamic system construction and simulation method provided by this example solves the "singular" phenomenon and process of the same object or the same particle moving in multiple or $\mathrm{N}$ directions simultaneously. The multi-rotation dynamic system simulation model gives consistent description and interpretation of the microscopic quantum effects and the spatio-temporal variations (bends) of the macroscopic universe.

\section{Quantum Entanglement}

The nature and characteristics of multiple-level dynamic systems show that any dynamic system or any dynamic system of a certain level is an entangled system. Multiple-level dynamic systems are multiple-level entangled systems. The individuals or subsystems in the entangled system are in the entangled state, and with the change of the dynamics of the entangled system, the entangled state between the individuals or subsystems also changes accordingly. For example, the Milky Way is an entangled dynamic system, in which stars, planets and interstellar matter are entangled in the rotation of the Milky Way; The solar system is an entangled dynamic system in the Milky Way, in which the sun, planets and interstellar matter are entangled in the rotation of the solar system. An atom is also an entangled dynamic system; in which protons, neutrons and even quarks, electrons, neutrinos, bosons and so on are entangled in the motion changes in the atom.

Being smaller than atoms, protons, neutrons and even elementary particles are also smaller entangled dynamic systems. Elementary particles differ greatly in mass or energy from one another. For example, if the mass of a proton is defined as 1, then the mass of an electron, electron neutrino, up quark, down quark, charm quark, top quark, muon, tau, etc. is $0.00054,<10^{-9}, 0.0047$, $0.0074,1.6,189,0.11$, and 1.9 times that of a proton [7], respectively. Elementary particles can transform into each other, especially during the annihilation of the positive and negative particles of elementary particles, which will generate other particles and release energy at the same time. It follows that elementary particles, or some elementary particles, have structures, that is, they can be subdivided.

An individual or subsystem in a dynamic system at any level is no longer entangled once it leaves the entangled system. Particles that leave the original entangled system are no longer entangled with each other, and continue to maintain the characteristics of the original entangled system, and the characteristics of the entangled system will disappear rapidly as these particles enter the new dynamic system and are subjected to any new force, and the mutual entanglement between particles does not exist. For example, if you separate two particles from an atom and transfer them to two places thousands of kilometers apart, there is no entanglement between them.

Since there is no entanglement between particles separated from the entangled system, it is natural that the teleportation will not occur between particles separated from the entangled system. In the famous EPR thought experiment [12], two separated particles are actually no longer entangled, and the "spooky super-distance action" of non-locality does not exist at all. If the characteristics of two particles are measured, only the remnants of the characteristics of the entangled system where the two particles were in the entangled state before separation can be obtained. 
On experimental measurements of quantum entanglement. In the experiment, the measurement of entangled particles must be "absolutely simultaneous" to obtain the entangled characteristics. According to the interpretation of quantum mechanics theory, due to the existence of "measurement collapse", if the entangled particles are not measured at the same time, the measurement results are not the properties or eigenvalues of the entangled particles. On the other hand, if the properties of particles not measured at exactly the same time point still show entanglement, it means that the two particles in the measurement are not entangled. The measurement results can only be the persistence or residual of the properties of the measured particles in the entangled state before they leave the entangled system, this indicates that the particles from the entangled system do not continue to be entangled. This proves that non-locality does not exist and that Einstein was right.

As for "absolute simultaneity", it is a theoretical concept that cannot be realized. Absolute simultaneity cannot be achieved in timing, even if the time difference between two particles measured is one infinite fraction of the speed of light, it is not absolutely simultaneous. For example, the detection devices that measure two particles that are disentangled from the system cannot achieve absolute simultaneous measurement because the connection lines between the detectors and the timer are infinitely different. So in the actual measurement, absolute simultaneity cannot be realized.

\section{The argument between Albert Einstein and Niels Bohr} [13] could be finished

They come to different conclusions or viewpoints from different perspectives or approaches to observe the quantum motion process (quantum effect). Bohr's conclusion was the result of direct observation of Figure 3 (c), that is, random, indeterminate, and superimposed. Einstein observed or reasoned from Figure 3 (d) or Figure 4 that "God does not play dice". They are both right and there is no contradiction in essence.

Bell's inequality $\left|P_{x z}-P_{z y}\right| \leq 1+P_{x y}$ cannot be the criterion for determining who was right between Einstein and Bohr. In fact, Bell's inequality is sometimes true and sometimes not, it's not a constant inequality. Whether the Bell's inequality is true or not depends on the structure of the multiple dynamic system in which the quantum is being measured and the direction of measurement.

7. Wave-particle duality of subatomic particles is a fundamental property of the periodic rotation motion of multilevel and multi-dimensional dynamic systems

The motion of microscopic particles in multidimensional dynamic systems has the characteristics of periodic rotational motion of it (the dynamic system), its motion is periodic and volatile in nature. The waveparticle duality of microscopic particles can be summarized as follows: first, a single microscopic particle has particle nature and its motion trajectory is fluctuating; Second, the microscopic particles behave as particles individually, but the swarm behaves as fluctuation. In short, microscopic particles are particles individually and are waves in groups.

\section{Chemical bond}

The atoms of the same multi-dimensional dynamical system interact with each other in the vortex motion, they are close to each other under the inertial forces of rotational motion, at the same time, the vortex energy quanta between them can also interact with electrons and other radioactive particles to create resistance to their proximity. The approaching force and the resistance force wind up to form a twisting force, which binds atoms to each other, and that's what bonds are all about.

\section{Dark matter and dark energy}

When the temperature of the object drops to $0 \mathrm{~K}$, the energetic particles around the vortex, such as protons, neutrons, and atoms, are almost dispersed, and they will achieve a closer bond between them. At this time, the material density of the object will increase, and at the same time, the energy will hardly radiate outward, so their existence cannot be observed from a long distance. Dark matter is something that doesn't emit radiation, and dark matter is also the way dark energy exists. The invisible quantum of energy in the universe is also how dark matter and dark energy exist when there is no radiation. All things in the universe operate in a multidimensional inertial system. The motion, change and interaction of all things in the universe are driven and maintained by different levels of inertial forces, and do not need dark matter or dark energy to drive or maintain.

\section{Mass}

The mass of an object is essentially the number of energieelements it contains. In the same inertial motion system, the mass of two objects can be measured and compared by the inertial mass. In everyday life and in scientific research, the mass of an object is replaced by the mass of inertia. The rest mass, motion mass and relativistic mass (total mass) are also different forms of mass. The gravitational mass is essentially the mass of inertia. Since any object is made up of energieelemetns, any object including all elementary particles has mass. 


\section{THE BASIC CHARACTERISTICS OF THE MULTI-LEVEL AND MULTI-DIMENSIONAL DYNAMIC SYSTEM}

In this embodiment, the motion model of the multilevel and multi-dimensional dynamic system constructed through simulation and construction can solve the technical problem that the motion model constructed in the existing technology cannot jointly explain the microscopic quantum effect and the macroscopic universe star galaxy. The motion model of the multi-level and multidimensional dynamic system constructed by it can play a very good role in the practical application and commercial value of the physical field:

1. The multi-level and multi-dimensional dynamic system model has important reference value to the research of physics and the innovation and development of theoretical physics.

2. The model of the multi-level and multi-dimensional dynamic system has enlightenment and appreciation value for the readers who are engaged in physics research and interested in physics and even other natural science.

3. Taking the multi-level and multi-dimensional dynamic system model as an experience project can enable people to personally experience the wonderful running process of high-dimensional space-time in the multi-dimensional dynamic system, which has high entertainment and commercial application value.

4. Unify the laws of motion of microscopic particles and macroscopic cosmic motion factors.

Above is only the preferred embodiment of the invention. It should be understood that the invention is not limited to the form disclosed herein; it should not be seen as an exclusion of other embodiments but as applicable to a variety of other combinations, modifications and contexts. And it should not be able to make changes within the scope of the ideas described in this article through the techniques or knowledge of the above teaching or related fields. The modifications and changes, made by personnel in the field and which do not deviate from the spirit and scope of the present invention, shall be within the protection scope of the claims attached to the present invention.

\section{FURTHER DISCUSSIONS}

\section{A.}

The techniques and methods of modeling the simulation of multiple dynamic systems have been established. As the multiplicity of multiple dynamic systems increases, the functional requirements of the computer will increase exponentially. If we want to realize multiple dynamic system simulation from micro quantum to macro universe, it is a huge challenge to the supercomputing power of the computer.

B.

The basic idea or path of the simulation technology and method of multiple dynamic systems has been established, but with the increase of the multiplicity of multiple dynamic systems, the mathematical description or description of the motion trajectory and iterative combination of multiple dynamic systems will be more and more difficult, which also poses new challenges to the mathematical description.

\section{C.}

The simulation of the distribution and movement of multiple dynamic systems in the universe will be another new field of future cosmic science research, which will reveal the basic structure and movement rules of the universe and all things.

\section{D.}

According to Einstein's principle of equivalence, inertial forces and gravitational forces are equivalent. If gravity is the inertial force, then the description or calculation of universal gravitation is the description or calculation of the expression of inertial force. If everything in the universe, including all stars and galaxies, were moving under inertial forces, then the super-massive compact object [14] at the center of our galaxy would be impossible to exist.

E.

The ordered motion of different quantities of energieelements forms different dynamic systems. Any dynamic system is an inertial system in rotational motion [1]. The power of any inertial motion system comes from the energieelements that make up the inertial system. At the most microscopic level, these inertial motion systems composed of different quantities of energieelements become the elementary particles of all classes. So far, scientists have identified and named 61 elementary particles . An ordered inertial motion system formed by different numbers of protons, neutrons, electrons, etc., is a variety of atoms. An inertial motion system formed by one or more atoms becomes molecules. By analogy, from planets to stars, to galaxies and clusters of galaxies are inertial motion systems of different levels in rotational motion. 
F.

Starting from energieelements, quanta, atoms, molecules, stars, star systems, galaxies and galaxy groups are all inertial motion systems of different levels composed of energieelements of high energy motion, and the inertial system of different levels has corresponding inertial forces. The strong and weak forces are the inertial forces (short-range forces) formed by microscopic particles; Electromagnetic force is the inertial force (long-range force) formed by the interaction between charged and magnetic objects. Gravity is the inertial force (remote force) formed by the inertial motion of macroscopic objects (stars and galaxies). Therefore, the four forces in the universe are essentially inertial forces, they are inertial forces of different levels of inertial motion systems.

\section{G.}

The structure of an atom is made up of multi-level and multiple dynamic systems. It can be inferred from the spectral lines of each element that each color light comes from the corresponding dynamic system in the atom, and the different color light must come from the electromagnetic radiation of different kinds or multiplicity of the dynamic system. Different colors of light also come from dynamic systems with different energy gradients. Since the energy gradient of the dynamic system with different kinds or multiplicity is discrete, the atomic spectral lines must also be discrete spectral fringes. Conversely, the spectral lines of elements indicate that the energy between different kinds or multiplicity of dynamic systems in atoms is quantized or discretized. The transfer of energy between different kinds or multiplicity of dynamical systems will absorb or radiate different energies of color light. The transformation between atomic internal dynamic systems is the essence of "electron transition" and the real process of "electron orbital transition". The energy reduction (by cooling) of dynamic systems of all kinds or multiple dynamic systems will change the structure of multi-level and multiple dynamic systems (increase in order, change in speed of motion, etc.), which is the fundamental reason for the change of material properties such as superconductivity or superflow.
[1] G. Yang and C. Yang, New Ideas and Research Directions for Multi-level and Multi-dimensional Dynamic Systems, OSF Preprints, doi:10.31219/osf.io/e3qpc (2020).

[2] G. Yang, A Multi-level and Multi-dimensional Dynamic Systems Model Construction and Simulation Method, State Intellectual Property Office of China, Patent Application No. 202011061206.7 (2020).

[3] G. Yang, Energy and the Universe, Nature and Science, 457-460 (2019).

[4] G. Hao, (2020), https://www.netpad.net.cn/ resource_web/course/\#/217337.

[5] G. Hao, (2020), https://www.netpad.net.cn/ presentationEditor/presentationPlay.html\#218962.

[6] G. Hao, (2020), https://www.netpad.net.cn/ resource_web/course/\#/219053.
[7] B. Greene, The fabric of the cosmos: Space, time, and the texture of reality (Knopf, 2004).

[8] S. Hawking, The universe in a nutshell (Bantam, 2001).

[9] J. B. Kaler, Heaven's touch: from killer stars to the seeds of life, how we are connected to the universe, (Princeton University Press, 2009).

[10] P. C. D. Davies and P. Davies, God and the new physics, (Simon and Schuster, 1984).

[11] B. Clegg, The God effect: Quantum entanglement, science's strangest phenomenon, (Macmillan, 2006)

[12] G. Guo, Subversion- Welcoming the Second Quantum Revolution, (Science Press, Beijing, 2022)

[13] Https://medium.com/cantors-paradise/the-bohreinstein-debate-baa0929a78b5.

[14] Https://www.nature.com/articles/s41567-020-01096-w 